IPMU-09-0006

\title{
Ghost condensate and generalized second law
}

\author{
Shinji Mukohyama \\ Institute for the Physics and Mathematics of the Universe (IPMU) \\ The University of Tokyo \\ 5-1-5 Kashiwanoha, Kashiwa, Chiba 27r7-8582, Japan
}

\begin{abstract}
Dubovsky and Sibiryakov recently proposed a scenario in which particles of different species propagate with different speeds due to their direct couplings to ghost condensate. It was argued that this extended version of ghost condensate allows a gedanken experiment leading to violation of the generalized second law. However, in the original ghost condensate scenario, difference in propagation speeds is suppressed by $M^{2} / M_{P l}^{2}$, where $M$ is the order parameter of spontaneous Lorentz breaking and $M_{P l}$ is the Planck scale. In this case the energy transfer necessary for the gedanken experiment is so slow that the timescale of decrease of entropy, if any, is always longer than the Jeans timescale of ghost condensate. Hence the generalized second law is not violated by the gedanken experiment in the original ghost condensate scenario. This conclusion trivially extends to gauged ghost condensation by taking into account accretion of gauged ghost condensate into a black hole.
\end{abstract}




\section{Introduction}

Precision observational data recently revealed that the expansion of our universe is accelerating. If Einstein's theory is correct, this requires that more than $70 \%$ of our universe is filled with invisible, negative pressure, energy. This energy is named dark energy, but we do not know what it really is. This situation reminds us of a story in the nineteenth century: when the perihelion shift of Mercury was discovered, some people hypothesized the existence of an invisible planet called Vulcan, a so-to-speak dark planet, to explain the anomalous behavior of Mercury. However, as we all know, the dark planet was not real and the correct explanation was to change gravity, from Newton's theory to Einstein's. With this in mind, it is probably natural to wonder if we can change Einstein's theory at long distances to address the mystery of dark energy.

From theoretical viewpoint, however, it is not easy to modify gravity in infrared (IR). For example, massive gravity [1] and DGP brane model [2] are known to have a ultraviolet (UV) scale at around $1000 \mathrm{~km}$, where effective field theories break down [3, 4]. This implies that those theories lose predictability at distances shorter than $1000 \mathrm{~km}$. For the DGP brane model in the branch without self-acceleration, nonlinear effects provide an extra contribution to the kinetic term of the longitudinal mode and push the UV scale to higher energy [5]. However, in the self-accelerating branch this contribution has a wrong sign and, thus, this branch includes a ghost.

Now let us remind ourselves of a situation with gauge field theory, hoping to find a hint. In gauge field theory, simply adding mass to a gauge boson changes the corresponding force law in IR but spoils its well-behaved properties in UV. It is the Higgs mechanism that is useful to modify the IR force law without ruining its UV behaviors. Indeed, the Higgs mechanism is integral to the standard model of particle physics and we are supposed to live in the Higgs phase of the theory.

Therefore, it seems promising to apply the idea of Higgs mechanism to gravity to modify general relativity in IR. Ghost condensation [6] is the simplest Higgs mechanism for gravity in the sense that it has only one Nambu-Goldstone boson 1. It opens up new type of gravitational phenomenologies such as IR modification of gravity [6], inflation with large non-Gaussianities [11, 12, 13], dark energy with $w<-1$ [14, 15, 16], rich non-linear dynamics [17], and so on.

The structure of the low energy effective field theory (EFT) of ghost condensation is determined by the symmetry breaking pattern as in the usual Higgs mechanism. We

\footnotetext{
${ }^{1}$ Closely related models include Lorentz-violating massive gravity [7, 8, Einstein aether theory 9 and gauged ghost condensation [10.
} 
assume that (i) derivative of a scalar field has a timelike constant vev, $\left\langle\partial_{\mu} \phi\right\rangle \neq 0$, and that (ii) the background spacetime metric is maximally symmetric, either Minkowski or de Sitter. By the assumption, the 4-dimensional spacetime diffeomorphism invariance is spontaneously broken down to the 3-dimensional spatial diffeomorphism invariance, i.e. the symmetry under $\vec{x} \rightarrow \vec{x}^{\prime}(t, \vec{x})$. Our strategy here is to write down the most general action invariant under this residual symmetry. After that, the action for the Nambu-Goldstone (NG) boson $\pi$ is obtained by undoing the unitary gauge. In Minkowski background, the result is

$$
L_{e f f}=M^{4}\left\{\frac{1}{2}\left(\dot{\pi}-\frac{1}{2} h_{00}\right)^{2}-\frac{\alpha}{M^{2}}\left(\vec{\nabla}^{2} \pi\right)^{2}+\cdots\right\},
$$

where $h_{00}$ is the time-time component of the metric perturbation, $M$ is the scale of symmetry breaking and $\alpha(>0)$ is a dimensionless constant of order unity. This low energy EFT is universal and should hold as far as the symmetry breaking pattern is the same. The scale $M$ is the order parameter of spontaneous Lorentz breaking and also plays the role of the UV cutoff scale of the low energy EFT. In the $M / M_{P l} \rightarrow 0$ limit, the $\pi$ sector is decoupled from gravity and general relativity is recovered. Also, proper analysis of scaling dimensions [6] shows that higher-dimensional operators indicated by dots in (1.1) are suppressed at least by some positive (but in general fractional) power of $E / M$, where $E$ represents the typical energy and/or momentum scale of the system.

If we include some of those higher-dimensional operators such as higher time derivative terms in (1.1), then new modes may appear but they are always outside the regime of validity of the EFT: they have frequencies of the order $M$ or higher. Therefore, as far as we are interested in physics at energies and momenta well below $M$, those extra modes are irrelevant. Moreover, properties of those high-frequency modes can be modified by other higher-dimensional operators without any noticeable changes to low energy physics. For these reasons we may and must concentrate on physics insensitive to those higher-dimensional operators, i.e. physics at energies and momenta well below $M$, unless we find a UV completion 2. Note that the current phenomenological bound on $M$ is [17]

$$
M \lesssim 100 \mathrm{GeV}
$$

The low energy effective action (1.1) plus the Einstein-Hilbert action exhibits IR modification of Einstein's theory in linearized gravitational potential [6]. The length

\footnotetext{
${ }^{2}$ See [18, 19] for some attempts towards a UV completion.
} 
scale $r_{J}$ and the timescale $t_{J}$ of the modification are given by

$$
r_{J} \sim \frac{M_{P l}}{M^{2}}
$$

and

$$
t_{J} \sim \frac{M_{P l}^{2}}{\sqrt{\alpha} M^{3}}
$$

Note that both scales are much longer than $1 / M$, provided that $M \ll M_{P l}$. These scales are analogous to the Jeans scale and thus we call them Jeans scales of ghost condensate.

Considering ghost condensation as a new candidate theory of gravity, it is important to test its consistency. Black hole thermodynamics [20] is probably useful for this purpose.

The purpose of this paper is to investigate whether the generalized second law of black hole thermodynamics holds in the presence of ghost condensate. A doubt was raised recently by Dubovsky and Sibiryakov [21. They proposed an extended version of ghost condensate in which particles of different species propagate with different speeds due to their direct couplings to ghost condensate. It was argued that Dubovsky-Sibiryakov's extension of ghost condensate allows a gedanken experiment leading to violation of the generalized second law. In this paper, on the contrary, we show that in the original ghost condensate scenario the generalized second law cannot be violated by the same gedanken experiment. The reason is that difference in propagation speeds is suppressed by $M^{2} / M_{P l}^{2}$, where $M$ is the order parameter of spontaneous Lorentz breaking and $M_{P l}$ is the Planck scale, since Lorentz invariance recovers in the limit $M^{2} / M_{P l}^{2} \rightarrow 0$. We shall of course take into account direct couplings generated by quantum corrections via gravitational interactions.

The rest of this paper is organized as follows. In Sec. 2 Dubovsky-Sibiryakov's extension of ghost condensate is described and it is shown that a gedanken experiment appears to violate the generalized second law in their theory. In Sec. 3 we go back to the original ghost condensate scenario and show that the same gedanken experiment cannot violate the generalized second law. In Sec. 4 we consider the gauged ghost condensate [10] and show the same result. Sec. 5] is devoted to a summary of this paper and some discussions.

\section{Dubovsky-Sibiryakov's extension}

Dubovsky and Sibiryakov [21] proposed an extension of the ghost condensate scenario by adding a specific direct coupling between the ghost condensate sector and matter 
fields. For a massless scalar field $\psi$, they added a derivative coupling to the ghost condensate sector as

$$
S_{\psi}=\int \sqrt{-g}\left[-\frac{1}{2} g^{\mu \nu} \partial_{\mu} \psi \partial_{\nu} \psi+\frac{1}{2 M_{D S}^{4}}\left(g^{\mu \nu} \partial_{\mu} \phi \partial_{\nu} \psi\right)^{2}\right] d^{4} x,
$$

where $\phi$ is the scalar field responsible for ghost condensate, $M_{D S}$ is some energy scale. Typically, $M_{D S}$ should be of order $M_{P l}$ but we leave it as free in this extension.

The massless field $\psi$ described by the action (2.1) propagates with the speed different from that inferred from the light cone structure of the metric $g_{\mu \nu}$. For example, in the Minkowski background $g_{\mu \nu}=\eta_{\mu \nu}$ with $\phi=M^{2} t$, it is easy to see that the propagation speed is not 1 but $(1+\epsilon)^{-1 / 2}$, where

$$
\epsilon=\frac{M^{4}}{M_{D S}^{4}}
$$

Since $M_{D S}$ is typically of order $M_{P l}, \epsilon$ is extremely small. Note that the known phenomenological upper bound on the order parameter of spontaneous Lorentz breaking in ghost condensate is (1.2). Nonetheless, we shall leave $\epsilon$ as a free parameter in this section.

For more general backgrounds with

$$
g^{\mu \nu} \partial_{\mu} \phi \partial_{\nu} \phi=-M^{4}
$$

the action (2.1) is rewritten as

$$
S_{\psi}=\int \sqrt{-g}\left[-\frac{1}{2} \tilde{g}^{\mu \nu} \partial_{\mu} \psi \partial_{\nu} \psi\right] d^{4} x=\int \sqrt{-\bar{g}}\left[-\frac{1}{2} \bar{g}^{\mu \nu} \partial_{\mu} \psi \partial_{\nu} \psi\right] d^{4} x
$$

where

$$
\begin{aligned}
\tilde{g}^{\mu \nu} & =-(1+\epsilon) u^{\mu} u^{\nu}+\left(g^{\mu \nu}+u^{\mu} u^{\nu}\right), \\
\tilde{g}_{\mu \nu} & =-\frac{1}{1+\epsilon} u_{\mu} u_{\nu}+\left(g_{\mu \nu}+u_{\mu} u_{\nu}\right), \\
\bar{g}_{\mu \nu} & =\frac{\sqrt{-g}}{\sqrt{-\tilde{g}}} \times \tilde{g}_{\mu \nu}, \\
\bar{g}^{\mu \nu} & =\frac{\sqrt{-\tilde{g}}}{\sqrt{-g}} \times \tilde{g}^{\mu \nu}
\end{aligned}
$$

and

$$
u_{\mu}=\frac{\partial_{\mu} \phi}{M^{2}}, \quad u^{\mu}=g^{\mu \nu} u_{\nu}
$$

Note that $g^{\mu \nu} u_{\mu} u_{\nu}=-1$. The field $\psi$ propagates along light cones of $\bar{g}_{\mu \nu}$ and its speed relative to $u^{\mu}$ is not 1 but again $(1+\epsilon)^{-1 / 2}$. 
For a timescale sufficiently shorter than the Jeans timescale (1.4), there is an approximate solution in Schwarzschild background [23]. The solution is as simple as

$$
\phi=M^{2} \tau
$$

where the Schwarzschild metric with the horizon radius $r_{g}$ is written in the Lemaitre reference frame as

$$
d s^{2}=-d \tau^{2}+\frac{r_{g} d R^{2}}{r(\tau, R)}+r^{2}(\tau, R) d \Omega^{2}, \quad r(\tau, R)=\left[\frac{3}{2} \sqrt{r_{g}}(R-\tau)\right]^{2 / 3} .
$$

This is not an exact solution but describes behavior of the system at most up to the Jeans timescale (1.4). This approximate solution indeed satisfies the condition (2.3). The corresponding effective metric $\bar{g}_{\mu \nu}$ for $\psi$ turns out to be a Schwarzschild metric with a different horizon radius $\bar{r}_{g}$,

$$
\left.d \bar{s}^{2}=-d \bar{\tau}^{2}+\frac{\bar{r}_{g} d \bar{R}^{2}}{\bar{r}(\bar{\tau}, \bar{R})}+\bar{r}^{2}(\bar{\tau}, \bar{R}) d \Omega^{2}, \quad \bar{r}(\bar{\tau}, \bar{R})=\left[\frac{3}{2}{\sqrt{\bar{r}_{g}}}_{(\bar{R}}-\bar{\tau}\right)\right]^{2 / 3},
$$

where

$$
\bar{r}_{g}=(1+\epsilon)^{5 / 4} r_{g}, \quad \bar{\tau}=(1+\epsilon)^{-1 / 4} \tau, \quad \bar{R}=(1+\epsilon)^{-1 / 4} R .
$$

Therefore, while the original Schwarzschild metric $d s^{2}$ has temperature $T_{b h}=\left(4 \pi r_{g}\right)^{-1}$, the $\psi$-metric $d \bar{s}^{2}$ has a different temperature

$$
T_{b h, \psi}=\frac{1}{4 \pi \bar{r}_{g}} \times \frac{d \bar{\tau}}{d \tau}=\frac{T_{b h}}{(1+\epsilon)^{3 / 2}} .
$$

Here, temperatures $T_{b h}$ and $T_{b h, \psi}$ are defined with respect to the original time variable $\tau$ at infinity and this is the reason why the factor $d \bar{\tau} / d \tau$ is included in the above expression for $T_{b h, \psi}$. Since different fields can have different $\epsilon$, a black hole can have different temperatures for different species.

The gedanken experiment in the Dubovsky-Sibiryakov extension consists of a Schwarzschild black hole with horizon radius $r_{g}$, two species $A$ and $B$ with $\epsilon_{A}>\epsilon_{B}$, and two spherical static shells surrounding the black hole each of which is made of $A$ and $B$, respectively. We denote temperatures of the black hole for $A$ and $B$ as $T_{b h, A}$ and $T_{b h, B}\left(T_{b h, A}<T_{b h, B}\right)$ and suppose that the shells of $A$ and $B$ have temperatures $T_{\text {shell, } A}$ and $T_{\text {shell,B}}$, respectively. One can tune the shells' temperatures so that

$$
T_{b h, A}<T_{\text {shell }, A}<T_{\text {shell,B }}<T_{b h, B}
$$

and that

$$
F_{\text {shell } \rightarrow b h, A}=-F_{\text {shell } \rightarrow b h, B}>0,
$$


where

$$
F_{\text {shell } \rightarrow b h, i}=\frac{\pi^{3} r_{g}^{2}}{15}\left[\Gamma_{i}\left(T_{\text {shell }, i}\right) T_{\text {shell }, i}^{4}-\Gamma_{i}\left(T_{b h, i}\right) T_{b h, i}^{4}\right] \quad(i=A, B)
$$

is the net flux of energy from the shell to the black hole for the species $i$ and $\Gamma_{i}(T)=$ $O(1)$ is a slowly varying function representing the gray body factor for the species $i$.

Note that the action (2.4) is exactly the same as the usual canonical action for a massless scalar field propagating in the corrected metric $d \bar{s}^{2}$ (not the original metric $d s^{2}$ ). Therefore, following the standard quantization procedure for a canonical scalar field in a fixed background geometry, we obtain the usual formula (2.14) for the net energy flux. Of course, treating the geometry $d \bar{s}^{2}$ as a fixed background is just an approximation. This approximation is justified if (i) the backreaction of Hawking radiation to the geometry is small and if (ii) relevant processes in the gedanken experiment are sufficiently faster than non-trivial dynamics of ghost condensate (such as Jeans-like instability and/or accretion into black hole). The condition (i) is satisfied if the black hole is sufficiently large. We shall investigate the condition (ii) in the next section.

To be more precise, (2.14) should be understood as the net flux of gravitational energy for each species $i$. Note that in the presence of direct couplings to ghost condensate, gravitational energy and particle-physics energy are not the same in general. For $\psi$ in flat background $\left(g_{\mu \nu}=\eta_{\mu \nu}\right.$ with $\left.\phi=M^{2} t\right)$, gravitational energy density, or $T_{00}$, is

$$
\rho_{\text {grav }}=T_{00}=\frac{1}{2}(1+3 \epsilon) \dot{\psi}^{2}+\frac{1}{2}(\vec{\nabla} \psi)^{2},
$$

while the particle-physics energy density, or the Hamiltonian density, is

$$
\rho_{\text {part }}=\mathcal{H}=\frac{1}{2}(1+\epsilon) \dot{\psi}^{2}+\frac{1}{2}(\vec{\nabla} \psi)^{2} .
$$

For small $\epsilon$, the difference is $O(\epsilon)$, as in (3.8) below.

In this setup, for a timescale sufficiently shorter than the Jeans timescale $t_{J}$, the black hole mass does not change. Energy is just transferred via the black hole from the shell of $A$ with lower temperature $T_{\text {shell, } A}$ to the shell of $B$ with higher temperature $T_{\text {shell,B}}$. Thus, the sum of entropies of two shells decreases. On the other hand, if we consider the horizon area (in the Planck unit) divided by four as black hole entropy then the black hole entropy does not change. In this way, the total entropy appears to decrease. Thus, this gedanken experiment in the Dubovsky-Sibiryakov extension appears to violate the generalized second law. 


\section{No violation of GSL in the original ghost condensate}

It is widely believed that the generalized second law should hold at least for quasistationary evolution of systems with black holes. In situations which have holographic descriptions, a black hole is dual to a thermal excitation and, thus, the generalized second law is dual to the ordinary second law of thermodynamics. Therefore, violation of the generalized second law would indicate lack of holographic descriptions [22]. For this reason, by demanding validity of the generalized second law, we may hope to exclude regions in the parameter space that do not allow holographic dual descriptions.

In the previous section we have reviewed the gedanken experiment in the DubovskySibiryakov extension of ghost condensate. However, we have not specified the scale $M_{D S}$ (and thus the value of $\epsilon$ ). Also, we have not taken into account two important time scales associated with the gedanken experiment. One is the Jeans time scale (1.4) of ghost condensate, and the other is the time scale in which shells' entropy would decrease. If the latter time scale is longer than the former then the gedanken experiment is invalidated. In this section we shall show that this is indeed the case in the original proposal of ghost condensation [6, 17]. In this case, excitations of ghost condensate become important before the gedanken experiment starts operating. Those excitations should accrete into the black hole and, accordingly, the total entropy is expected to increase.

In the original ghost condensate the scale $M$ is the order parameter of spontaneous Lorentz breaking. Therefore, Lorentz invariance should recover in the limit $M^{2} / M_{P l}^{2} \rightarrow 0$ limit. In particular, $\epsilon$ should vanish in this limit and thus we have

$$
\epsilon=O\left(\frac{M^{2}}{M_{P l}^{2}}\right) .
$$

Note that the typical value of $M_{D S}$ is of order $M_{P l}$ and leads to $\epsilon=O\left(M^{4} / M_{P l}^{4}\right)$. This corresponds to the typical strength of direct couplings generated by quantum corrections via gravitational interactions. The condition (3.1) is less restrictive than this, but is still more than sufficient to protect the generalized second law.

By setting

$$
\epsilon_{i}=O\left(\frac{M^{2}}{M_{P l}^{2}}\right),
$$

we obtain

$$
T_{b h, i}=T_{b h} \times\left[1+O\left(\epsilon_{i}\right)\right],
$$


and

$$
T_{b h, B}-T_{b h, A}=T_{b h} \times O\left(\frac{M^{2}}{M_{P l}^{2}}\right) .
$$

Since $T_{\text {shell }, i}$ are bounded from below and from above by $T_{b h, A}$ and $T_{b h, B}$, respectively as in (2.12), it also follows that

$$
T_{\text {shell }, i}-T_{b h, i}=T_{b h} \times O\left(\frac{M^{2}}{M_{P l}^{2}}\right) .
$$

This implies that the net flux of energy for each species is

$$
\left|F_{\text {shell } \rightarrow b h, i}\right| \sim r_{h}^{2} T_{b h}^{3} \times\left|T_{\text {shell }, i}-T_{b h, i}\right|=T_{b h}^{2} \times O\left(\frac{M^{2}}{M_{P l}^{2}}\right) .
$$

Since the heat transfer is suppressed by $M^{2} / M_{P l}^{2}$, the rate of decrease of shells' entropy is also suppressed. Of course, if we could wait for infinite time then the gedanken experiment would still lead to violation of the generalized second law. However, as already stated, the setup of the gedanken experiment does not persist forever but can last only up to the Jeans timescale (1.4) at most. Thus, under the equi-flux condition (2.13), let us estimate the maximum decrease of shells' entropy which the gedanken experiment of this sort could in principle lead to within the Jeans timescale $t_{J}$ :

$$
\begin{aligned}
\left|\Delta S_{\text {shells }}\right|_{\text {max }} & =\left|\frac{d S_{\text {shell }, A}}{d t}+\frac{d S_{\text {shell }, B}}{d t}\right| \times t_{J} \\
& =F_{\text {shell } \rightarrow \text { bh,A }} \times\left(\frac{1}{T_{\text {shell }, A}} \cdot \frac{d E_{\text {part }, A}}{d E_{\text {grav }, A}}-\frac{1}{T_{\text {shell }, B}} \cdot \frac{d E_{\text {part }, B}}{d E_{\text {grav }, B}}\right) \times t_{J} \\
& \sim O\left(\frac{M^{2}}{M_{P l}^{2}}\right) \times \frac{F_{\text {shell } \rightarrow \text { bh, }, A}}{T_{b h}} \times t_{J} \\
& \sim O\left(\frac{M^{2}}{M_{P l}^{2}}\right) \times \frac{T_{b h}}{M},
\end{aligned}
$$

where

$$
\frac{d E_{\text {part }, i}}{d E_{\text {grav }, i}}=1+O\left(\epsilon_{i}\right)
$$

is the ratio of particle-physics energy to gravitational energy for the species $i$.

Validity of the effective field theory requires that the black hole temperature be lower than the UV cutoff scale $M$ :

$$
T_{b h} \ll M \text {. }
$$

Equivalently, the horizon radius $r_{g}$ must be sufficiently greater than $1 / M$. Otherwise, we cannot trust the low energy effective field theory and cannot justify the gedanken 
experiment at all. Under this condition, the maximum decrease of shells' entropy (3.7) is bounded as

$$
\left|\Delta S_{\text {shells }}\right|_{\text {max }} \ll O\left(\frac{M^{2}}{M_{P l}^{2}}\right) \ll O(1) .
$$

Therefore, shells' entropy cannot decrease even by $O\left(M^{2} / M_{P l}^{2}\right)$ within the Jeans timescale of ghost condensate. In other words, the timescale of decrease of shells' entropy, if any, is always longer than the maximum timescale for which the setup of the gedanken experiment can in principle last.

In conclusion, the gedanken experiment suggested in [21] does not violate the generalized second law in the original ghost condensation scenario. The essential reason for this is that difference in propagation speeds is suppressed by $M^{2} / M_{P l}^{2}$, where $M$ is the order parameter of spontaneous Lorentz breaking and $M_{P l}$ is the Planck scale, since Lorentz invariance recovers in the limit $M^{2} / M_{P l}^{2} \rightarrow 0$. This makes the semiclassical heat flow so slow that shells' entropy cannot decrease before accretion of ghost condensate induced by the Jeans instability increases black hole entropy.

\section{Gauged ghost condensate}

As another example, let us consider gauged ghost condensation [10]. In this case, if the gauge coupling is large enough then the Jeans instability disappears. Nonetheless, there still is the maximum time scale in which the gedanken experiment can in principle last. In the presence of the higher derivative term, the black hole solution (2.7) is just an approximate solution. Actually, gauged ghost condensate slowly

gets excited and those excitations accrete towards the black hole. As a result, the black hole entropy increases. The time scale of accretion $t_{a c c}$ can be estimated from eq. (6.13) of [10]. The result is

$$
t_{a c c} \sim \frac{m_{b h} M_{P l}}{M^{3}} \sim t_{J} \times \frac{M_{P l}}{T_{b h}}
$$

where we have defined $t_{J}$ by (1.4). Since $T_{b h} \ll M \ll M_{P l}$ is required for validity of the effective field theory, we have $t_{a c c} \gg t_{J}$. Thus, the absence of Jeans instability makes it possible for the gedanken experiment in the gauged ghost condensate to last longer than in the (ungauged) ghost condensate.

Nonetheless, assuming again that $\epsilon_{i}=O\left(M^{2} / M_{P l}^{2}\right)$ and repeating the analysis in the previous section, we still obtain

$$
\left|\Delta S_{\text {shells }}\right|_{\text {max }} \sim O\left(\frac{M}{M_{P l}}\right) \ll O(1) .
$$


In other words, black hole entropy increases due to accretion before DubovskySibiryakov's gedanken experiment starts operating. Thus, the gedanken experiment does not lead to violation of the generalized second law in gauged ghost condensation.

\section{Summary and discussion}

We have revisited the gedanken experiment suggested by Dubovsky and Sibiryakov [21]. In their extension of ghost condensate, the gedanken experiment appears to violate the generalized second law. This result may imply lack of holographic dual descriptions for their extension 22 .

Dubovsky-Sibiryakov's extension requires difference in propagation speeds for different species. In the limit where Lorentz invariance is recovered, the generalized second law should recover.

In the original ghost condensate scenario, difference in propagation speeds is suppressed by $M^{2} / M_{P l}^{2}$, where $M$ is the order parameter of spontaneous Lorentz breaking due to ghost condensation and $M_{P l}$ is the Planck scale. This is because Lorentz invariance recovers in the limit $M^{2} / M_{P l}^{2} \rightarrow 0$. For this reason, the energy transfer necessary for the gedanken experiment is so slow that the timescale of decrease of shells' entropy, if any, is always longer than the Jeans timescale of ghost condensate. The latter is the maximum timescale for which the setup of the gedanken experiment can in principle last. Therefore, the gedanken experiment does not lead to violation of the generalized second law in the original ghost condensation scenario. Note that the Jeans timescale is longer than the present age of the universe if $M \lesssim 10 \mathrm{MeV}$. Even in this case the Jeans time is not long enough for the gedanken experiment.

We have also shown a similar result for the gauged ghost condensation. In this case, we have considered the time scale of black hole accretion and shown that the gedanken experiment cannot decrease the total entropy within this time scale. It is also possible to apply this consideration to the ungauged ghost condensation since the accretion rate is the same. However, the argument in Sec. 3 based on Jeans timescale suffices in the ungauged case.

Jeans instability of ghost condensate disappears in de Sitter background if $H \gtrsim$ $1 / t_{J}$, where $H$ is the Hubble expansion rate and $t_{J}$ is the Jeans timescale. Hence, one might expect that the setup of the gedanken experiment could last longer than $t_{J}$ in asymptotically de Sitter backgrounds, say Schwarzschild-de Sitter background. However, this is most likely too naive. The essential reason for the absence of Jeans instability in a pure de Sitter background (with large enough $H$ ) is that the vev $\left\langle\partial_{\mu} \phi\right\rangle$ has a positive expansion. On the other hand, in a Schwarzschild-de Sitter background, 
the vev $\left\langle\partial_{\mu} \phi\right\rangle$ has a negative expansion (i.e. it is contracting) near the black hole, while it has a positive expansion far enough from the black hole. Therefore, while the Jeans instability can disappear in the far region, it should exist near the black hole. In particular, in the intermediate region where the expansion almost vanishes, the Jeans instability should be essentially the same as that in flat background. For this reason, in either asymptotically flat or asymptotically de Sitter background, the Jeans timescale estimated in Minkowski background is the maximum timescale for which the setup of the gedanken experiment can in principle last. Therefore, even in asymptotically de Sitter background, the gedanken experiment does not violate the generalized second law in the original ghost condensate scenario.

Now let us discuss extensions of the gedanken experiment to get some insights about UV completion of ghost condensate.

So far, we have considered only two species $A$ and $B$ in the gedanken experiment. What happens if we consider many species with different $\epsilon_{i}$ and thus with different propagation speeds? Of course, only light degrees of freedom can contribute to the gedanken experiment: they must be sufficiently lighter than $T_{b h}$. Based on the gedanken experiment with many light degrees of freedom, we conjecture that

$$
\frac{1}{\sqrt{\alpha}} \sum_{1 \leq i<j \leq N}\left(\epsilon_{i}-\epsilon_{j}\right)^{2}=O\left(\frac{M^{2}}{M_{P l}^{2}}\right)
$$

should be satisfied by a sensible UV completion. Here, $M$ is the order parameter of ghost condensation, $\alpha(>0)$ is the dimensionless coefficient of the relevant higher derivative term (see (1.1) ) and $N$ is the number of light fields whose mass is well below $M$. If this conjecture is correct then the extended gedanken experiment does not violate the generalized second law.

Next let us consider extension involving ghost condensate quanta. So far, we have treated ghost condensate as a fixed background and have not considered its excitation $\pi(\propto \delta \phi)$. A natural question now is "what happens if we replace the species $A$ or $B$ in Dubovsky-Sibiryakov's gedanken experiment by $\pi$ ?" To address this question let us first consider how a black hole radiates $\pi$ quanta in the absence of higher-derivative terms. Since the rest frame of the ghost condensate background is in-falling towards the black hole and $\pi$ has a vanishing sound speed, $\pi$ quanta cannot escape to infinity. This means that there would be no radiation of $\pi$ quanta from a black hole in the absence of higher-derivative terms. In reality, higher-derivative terms are present and a black hole radiates $\pi$ quanta. The spectrum of Hawking radiation of $\pi$ is still highly suppressed and non-thermal [24] although the result seems UV sensitive. From this, one might naively guess that by replacing the species $A$ (the one with lower temperature) in the gedanken experiment by $\pi$, the generalized 
second law could be violated. However, this is not necessarily true. In order to start this modified gedanken experiment, we need to prepare a quasi-static shell made of thermally excited $\pi$ quanta. The precise way the shell is prepared may be UV sensitive (because of e.g. formation of caustics [17]) but should be through gravity anyway. Let us assume that a quasi-static shell is somehow prepared and ask how it radiates. In the absence of higher-derivative terms, it would not radiate $\pi$ quanta since $\pi$ has vanishing sound speed. In the presence of higher-derivative terms, the thermal shell of $\pi$ can radiate but the spectrum of $\pi$ radiation is most likely nonthermal and highly suppressed. We now conjecture that a sensible UV completion should be such that the spectrum of $\pi$ radiation from a black hole and the spectrum from a thermal shell of $\pi$ with the same temperature are essentially identical 3 . If this conjecture is correct then energy flows stop when all three temperatures agree and the total entropy is maximized. Thus the conjecture is sufficient to prevent the generalized second law from being violated by this modified gedanken experiment. Note that this conjecture does not contradict with any known facts. While the proof (or disproof) of this conjecture requires a concrete setup for UV completion and is beyond the scope of the present paper, it is appropriate to state it as a conjecture since there is no known contradiction.

In this paper we have not considered yet-another gedanken experiment suggested by Eling, et al. [25]. It is based on a purely classical process analogous to the Penrose process [26, 27]. They mainly considered Einstein aether theory [9] but the process could in principle be applied to Dubovsky-Sibiryakov's extension of ghost condensate (with relatively large $\left|\epsilon_{i}\right|$ ). They concluded that the generalized second law can be violated in these theories. However, in the original ghost condensate scenario, $\left|\epsilon_{i}\right|$ is generated by quantum corrections and thus suppressed by $M^{2} / M_{P l}^{2}$. In this case one can show that the analogue of the Penrose process is kinematically forbidden unless particles are initially released from positions extremely close to the horizon. The essential reason for this is that the "ergo-region" disappears in the $\epsilon_{i} \rightarrow 0$ limit. This kinematical fact means that particles just before being released are accelerated rather strongly and quantum effects such as buoyancy force due to the thermal bath near the horizon may become important. Actually, as Unruh and Wald showed in [28, 29] (see also [30, 31].), the buoyancy force is essential for recovery of the generalized second law in a gedanken experiment in general relativity. At the very least, it is probably fair to say that we should include quantum effects consistently 4 . Also, we may have

\footnotetext{
${ }^{3}$ Difference in the two spectra is allowed to the extent that the timescale of decrease of total entropy, if any, is longer than either the Jeans timescale of ghost condensate or the lifetime of the quasi-static shell.

${ }^{4}$ If we do not take into account quantum effects at all then $\epsilon_{i}=0$ in the original ghost condensate
} 
to take into account the fact that not only the analogue of the Penrose process but also normal processes are allowed in the "ergo-region". These normal processes of course include e.g. soft elastic scattering and can dominate over the analogue of the Penrose process, especially in the thin "ergo-region" limit. Careful consideration of these issues is an interesting future subject but beyond the scope of this paper.

Excitations of ghost condensate can carry not only positive energy but also negative energy. By sending negative energy to a black hole, one might hope to violate the generalized second law [32]. However, the process considered in [32] does not satisfy basic conservation laws. Gravitational energy of excitation of ghost condensate is the sum of particle-physics energy and the charge associated with shift symmetry [6]. The particle-physics energy is non-negative and the shift charge is conserved. Hence, excitations with negative gravitational energy are always accompanied by excitations with larger positive energy. Therefore, if a negative energy falls into a black hole then more positive energy follows after that. This means that the black hole entropy should increase after all, if the conservation law is properly taken into account.

\section{Note added}

In Sec. 5 we have briefly commented on a yet-another gedanken experiment suggested by Eling, et al. [25]. In ref. [33] it was shown that efficiency of the gedanken experiment is highly suppressed by the factor $M^{2} / M_{P l}^{2}$ and that it is always lower than accretion of ghost condensate into a black hole. For this reason, the gedanken experiment suggested by Eling, et al. [25] does not violate the generalized second law in ghost condensate. In Sec. 5 we have also commented on negative energy carried by excitations of ghost condensate. In this respect, ref. [33] proved an averaged null energy condition, which prevents the negative energy from violating the generalized second law in a coarse-grained sense.

\section{Acknowledgements}

The author would like to thank Hsin-Chia Cheng, Sergei Dubovsky, Andrei Linde, Sergey Sibiryakov, Takahiro Tanaka and Aron Wall for useful comments. The work of the author was supported in part by MEXT through a Grant-in-Aid for Young Scientists (B) No. 17740134, by JSPS through a Grant-in-Aid for Creative Scientific Research No. 19GS0219, and by the Mitsubishi Foundation. This work was supported

scenario and there is no paradox in the first place. 
by World Premier International Research Center InitiativeWPI Initiative), MEXT, Japan.

\section{References}

[1] M. Fierz and W. Pauli, "On relativistic wave equations for particles of arbitrary spin in an electromagnetic field," Proc. Roy. Soc. Lond. A 173, 211 (1939).

[2] G. R. Dvali, G. Gabadadze and M. Porrati, "4D gravity on a brane in 5D Minkowski space," Phys. Lett. B 485, 208 (2000) arXiv:hep-th/0005016].

[3] N. Arkani-Hamed, H. Georgi and M. D. Schwartz, "Effective field theory for massive gravitons and gravity in theory space," Annals Phys. 305, 96 (2003) arXiv:hep-th/0210184.

[4] M. A. Luty, M. Porrati and R. Rattazzi, "Strong interactions and stability in the DGP model," JHEP 0309, 029 (2003) arXiv:hep-th/0303116.

[5] A. Nicolis and R. Rattazzi, "Classical and quantum consistency of the DGP model," JHEP 0406, 059 (2004) arXiv:hep-th/0404159.

[6] N. Arkani-Hamed, H. C. Cheng, M. A. Luty and S. Mukohyama, "Ghost condensation and a consistent infrared modification of gravity," JHEP 0405, 074 (2004) arXiv:hep-th/0312099.

[7] V. A. Rubakov, "Lorentz-violating graviton masses: Getting around ghosts, low strong coupling scale and VDVZ discontinuity," arXiv:hep-th/0407104.

[8] S. L. Dubovsky, "Phases of massive gravity," JHEP 0410, 076 (2004) arXiv:hep-th/0409124.

[9] T. Jacobson and D. Mattingly, "Gravity with a dynamical preferred frame," Phys. Rev. D 64, 024028 (2001) arXiv:gr-qc/0007031.

[10] H. C. Cheng, M. A. Luty, S. Mukohyama and J. Thaler, "Spontaneous Lorentz breaking at high energies," JHEP 0605, 076 (2006) [arXiv:hep-th/0603010].

[11] N. Arkani-Hamed, P. Creminelli, S. Mukohyama and M. Zaldarriaga, "Ghost Inflation," JCAP 0404, 001 (2004) [arXiv:hep-th/0312100].

[12] L. Senatore, "Tilted ghost inflation," Phys. Rev. D 71, 043512 (2005) arXiv:astro-ph/0406187. 
[13] C. Cheung, P. Creminelli, A. L. Fitzpatrick, J. Kaplan and L. Senatore, "The Effective Field Theory of Inflation," JHEP 0803, 014 (2008) arXiv:0709.0293 [hep-th]].

[14] P. Creminelli, M. A. Luty, A. Nicolis and L. Senatore, "Starting the universe: Stable violation of the null energy condition and non-standard cosmologies," JHEP 0612, 080 (2006) arXiv:hep-th/0606090.

[15] S. Mukohyama, "Accelerating universe and cosmological perturbation in the ghost condensate," JCAP 0610, 011 (2006) arXiv:hep-th/0607181.

[16] P. Creminelli, G. D'Amico, J. Norena and F. Vernizzi, "The Effective Theory of Quintessence: the wi-1 Side Unveiled," arXiv:0811.0827 [astro-ph].

[17] N. Arkani-Hamed, H. C. Cheng, M. A. Luty, S. Mukohyama and T. Wiseman, "Dynamics of Gravity in a Higgs Phase," JHEP 0701, 036 (2007) arXiv:hep-ph/0507120].

[18] M. L. Graesser, I. Low and M. B. Wise, "Towards a high energy theory for the Higgs phase of gravity," Phys. Rev. D 72, 115016 (2005) arXiv:hep-th/0509180].

[19] S. Mukohyama, "Towards a Higgs phase of gravity in string theory," JHEP 0705, 048 (2007) arXiv:hep-th/0610254.

[20] For example, S. Mukohyama, "The origin of black hole entropy," arXiv:gr-qc/9812079; and references therein.

[21] S. L. Dubovsky and S. M. Sibiryakov, "Spontaneous breaking of Lorentz invariance, black holes and perpetuum mobile of the 2nd kind," Phys. Lett. B 638, 509 (2006) arXiv:hep-th/0603158.

[22] N. Arkani-Hamed, S. Dubovsky, A. Nicolis, E. Trincherini and G. Villadoro, "A Measure of de Sitter Entropy and Eternal Inflation," JHEP 0705, 055 (2007) arXiv:0704.1814 [hep-th]].

[23] S. Mukohyama, "Black holes in the ghost condensate," Phys. Rev. D 71, 104019 (2005) arXiv:hep-th/0502189.

[24] B. Feldstein, "Hawking Radiation in the Ghost Condensate is Non-Thermal," Phys. Rev. D 78, 064061 (2008) [arXiv:0807.0253 [hep-th]].

[25] C. Eling, B. Z. Foster, T. Jacobson and A. C. Wall, Phys. Rev. D 75, 101502 (2007) arXiv:hep-th/0702124. 
[26] R. Penrose, "Gravitational collapse: The role of general relativity," Riv. Nuovo Cim. 1, 252 (1969) [Gen. Rel. Grav. 34, 1141 (2002)].

[27] R. Penrose and R. M. Floyd, "Extraction of rotational energy from a black hole," Nature 229, 177 (1971).

[28] W. G. Unruh and R. M. Wald, "Acceleration Radiation And Generalized Second Law Of Thermodynamics," Phys. Rev. D 25, 942 (1982).

[29] W. G. Unruh and R. M. Wald, "Entropy Bounds, Acceleration Radiation, And The Generalized Second Law," Phys. Rev. D 27, 2271 (1983).

[30] T. Shimomura and S. Mukohyama, "Does the generalized second law require entropy bounds for a charged system?," Phys. Rev. D 61, 064020 (2000) arXiv:gr-qc/9906047.

[31] S. Gao and R. M. Wald, "The "physical process" version of the first law and the generalized second law for charged and rotating black holes," Phys. Rev. D 64, 084020 (2001) arXiv:gr-qc/0106071.

[32] B. Feldstein, "Spontaneous Lorentz Violation, Negative Energy and the Second Law of Thermodynamics," arXiv:0904.1212 [hep-th].

[33] S. Mukohyama, "Can ghost condensate decrease entropy?," arXiv:0908.4123 [hep-th]. 